\title{
Grau de conhecimento sobre lesões potencialmente malignas e câncer de boca e autopercepção da qualidade de vida em idosos
}

\author{
Degree of knowledge about potentially malignant lesions and oral cancer and self-perceived quality \\ of life in the elderly
}

Grado de conocimiento sobre lesiones potencialmente malignas y cáncer bucal y calidad de vida autopercibida en ancianos

Recebido: 15/07/2021 | Revisado: 21/07/2021 | Aceito: 25/07/2021 | Publicado: 01/08/2021

\author{
Soraya Maria Farias Sicsu \\ ORCID: https://orcid.org/0000-0002-2843-3356 \\ Universidade Federal do Amazonas, Brasil \\ E-mail: sorayasicsu@gmail.com \\ Danielle Rodrigues Carvalho da Costa \\ ORCID: https://orcid.org/0000-0003-3768-9561 \\ Universidade Federal do Amazonas, Brasil \\ E-mail: daniellecarvalho824@gmail.com \\ Stella Maria Farias Sicsu \\ ORCID: https://orcid.org/0000-0002-2198-813X \\ Universidade Federal do Amazonas, Brasil \\ E-mail: stellasicsu7@gmail.com \\ José Eduardo Gomes Domingues \\ ORCID: https://orcid.org/0000-0002-8926-7830 \\ Universidade Federal do Amazonas, Brasil \\ E-mail: jedomingues@ufam.edu.br \\ Geisy Rebouças Lima Brasil \\ ORCID: https://orcid.org/0000-0002-9200-6375 \\ Universidade Federal do Amazonas, Brasil \\ E-mail: geisylima@hotmail.com \\ Nikeila Chacon de Oliveira Conde \\ ORCID: https://orcid.org/0000-0002-3615-6328 \\ Universidade Federal do Amazonas, Brasil \\ E-mail: nikeilaconde@ufam.edu.br
}

\begin{abstract}
Resumo
Objetivos: Verificar o conhecimento acerca das lesões potencialmente malignas, avaliar a condição de saúde bucal quanto à presença de lesões potencialmente malignas e avaliar a qualidade de vida. Metodologia: Estudo transversal com amostra de 108 idosos, com aplicação de questionários sobre o conhecimento das lesões potencialmente malignas, câncer de boca, autoexame, qualidade de vida e realização do exame clínico. Resultados: Dos idosos, 3,7\% souberam o que é câncer bucal e 38,9\% ouviram/leram sobre lesões potencialmente malignas. Detectaram-se lesões em 1,8\% dos idosos. Não houve diferenças significantes no índice GOHAI. Correlacionando a presença destas lesões ao conhecimento, dos indivíduos sem lesões, 46,2\% sabem o que é câncer bucal, os que apresentaram lesões negaram conhecer o assunto. Além disso, $100 \%$ da amostra declarou ter conhecimento que o álcool/tabaco se relacionam ao câncer bucal e $88,7 \%$ do grupo de indivíduos que não possuíam lesões potencialmente malignas também concordaram com esta afirmação. Conclusão: Constatou-se um baixo grau de conhecimento e prevalência de lesões bucais potencialmente malignas. A qualidade de vida não teve influência quanto à presença ou ausência de lesões. Dos idosos pesquisados que apresentaram lesões, nenhum soube dizer o que é câncer bucal e 50\% dos que não possuíam lesões bucais demonstraram entendimento sobre estas desordens. Assim, o desenvolvimento de maiores ações de promoção e prevenção da saúde bucal geriátrica, vinculada a esclarecimentos quanto às lesões potencialmente malignas e o câncer de boca, são notavelmente imprescindíveis.
\end{abstract}

Palavras-chave: Ferimentos e lesões; Neoplasias bucais; Odontologia geriátrica; Qualidade de vida; Autoexame.

\section{Abstract}

Objectives: To verify the knowledge about potentially malignant lesions, to evaluate the oral health condition regarding the presence of potentially malignant lesions and to evaluate the quality of life. Methods: Cross-sectional study with a sample of 108 elderly people, with the application of questionnaires about the knowledge of potentially malignant lesions, mouth cancer, self-examination, quality of life and clinical examination. Results: Of the elderly, 
$3.7 \%$ knew what oral cancer is and 38.9\% heard / read about potentially malignant lesions. Injuries were detected in $1.8 \%$ of the elderly. There were no significant differences in the GOHAI index. Correlating the presence of these injuries to the knowledge, of the individuals without injuries, $46.2 \%$ know what oral cancer is, those who presented injuries denied knowing the subject. In addition, $100 \%$ of the sample declared to have knowledge that alcohol/tobacco is related to oral cancer and $88,7 \%$ of the group of individuals who did not have potentially malignant lesions also agreed with this statement. Conclusion: There was a low level of knowledge and prevalence of potentially malignant oral lesions. Quality of life did not influence the presence or absence of injuries. Of the elderly people surveyed who had lesions, none could say what oral cancer is and 50\% of those who did not have oral lesions demonstrated understanding about these disorders. Thus, the development of greater actions for the promotion and prevention of geriatric oral health, linked to clarifications regarding potentially malignant lesions and oral cancer, are remarkably essential.

Keywords: Wounds and injuries; Oral neoplasms; Geriatric dentistry; Quality of life; Self exam.

\section{Resumen}

Objetivos: Verificar el conocimiento sobre lesiones potencialmente malignas, evaluar el estado de salud bucal com respecto a la presencia de lesiones potencialmente malignas y evaluar la calidad de vida. Métodos: Estudio transversal con uma muestra de 108 ancianos, con aplicación de cuestionarios sobre conocimiento de lesiones potencialmente malignas, cáncer bucal, autoexamen, calidad de vida y examen clínico. Resultados: De los ancianos, el 3,7\% sabía qué es el cáncer oral y el 38,9\% escuchó/leyó sobre lesiones potencialmente malignas. Se detectaron lesiones en el 1,8\% de los ancianos. No hubo diferencias significativas em el índice GOHAI. Correlacionando la presencia de estas lesiones com el conocimiento de los indivíduos sin lesiones, el 46,2\% sabe qué es el cáncer bucal, los que presentam lesiones niegam conocer el tema. Además, el 100\% de la muestra declaró tener conocimiento de que el alcohol/tabaco está relacionado con el cáncer oral y el $88,7 \%$ del grupo de indivíduos que no presentaban lesiones potencialmente malignas también estuvo de acuerdo com esta afirmación. Conclusión: Existía um bajo nível de conocimiento y prevalencia de lesiones orales potencialmente malignas. La calidad de vida no influyó en la presencia o ausencia de lesiones. De los ancianos encuestados que tenían lesiones, ninguno pudo decir qué es el cáncer oral y el $50 \%$ de los que no tenían lesiones orales demostraron comprensión sobre estos transtornos. Así, el desarrollo de mayores acciones para la promoción y prevención de la salud bucal geriátrica, ligadas a aclaraciones sobre las lesiones potencialmente malignas y el cáncer bucal, resultan de vital importancia.

Palabras clave: Heridas y traumatismos; Neoplasias bucales; Odontología geriátrica; Calidad de vida; Autoexamen.

\section{Introdução}

O câncer de boca representa uma doença maligna que afeta lábios, estruturas da boca, como gengivas, mucosa jugal, palato duro, língua (principalmente bordos) e soalho bucal. É mais comum em homens acima dos 40 anos e a maioria dos casos é diagnosticada em estágios avançados (INCA, 2021). Estimou-se que a incidência de neoplasias malignas na cavidade bucal no estado do Amazonas no ano de 2020 seria de 6,85 casos para cada 100 mil homens e de 1,99 casos para cada 100 mil mulheres (INCA, 2020). O câncer constitui um problema de saúde pública mundial: de cada sete mortes no mundo, uma devese ao câncer, sendo mais que HIV/AIDS, tuberculose e malária juntos (Braz et al., 2018).

As lesões potencialmente malignas são alterações teciduais que podem se transformar em tumor maligno a qualquer momento, mas também podem permanecer no estágio não maligno por tempo indefinido, e até mesmo nunca se transformar, principalmente se forem evitados estímulos nocivos sobre o tecido já alterado Dentre as classificações das lesões potencialmente malignas, as mais expressivas são as leucoplasia, eritroplasia e queilite actínica. Os desenvolvimentos destas lesões podem estar relacionados ao uso de álcool e tabaco, exposição contínua à radiação solar, traumatismos crônicos, estado nutricional, vírus, imunodeficiência, fatores genéticos e ambientais. (Kignel, 2013).

Numerosos distúrbios foram associados a um risco aumentado do carcinoma de células escamosas, incluindo a leucoplasia, eritroplasia, líquen plano oral, fibrose submucosa oral, queilite actínica, lesões palatinas, fumar charuto, lúpus eritematoso discóide e algumas doenças hereditárias. Do ponto de vista clínico, a grande maioria das lesões apresentando manchas brancas, com ou sem áreas avermelhadas, são as mais prováveis de terem um diagnóstico inespecífico. Embora esses distúrbios tenham um risco estatístico aumentado de malignidade, é muito difícil prever o resultado para um paciente individual. (Speight; Khurram; Kujan, 2018). 
É possível observar que a distribuição epidemiológica do câncer no Brasil sugere uma transição epidemiológica em andamento, decorrente principalmente da redefinição, no último século, do padrão de vida do brasileiro, com maior uniformização das condições de trabalho, nutrição e consumo, o que gerou aumento da expectativa de vida e envelhecimento populacional (Cavalcante et al., 2016). A população idosa cresce rapidamente no Brasil, passando de 13,8\% em 2020, para $33,7 \%$ em 2060. Nosso país será a sexta população mais idosa do mundo, com aproximadamente $15 \%$ da população total em 2025 (IBGE, 2010).

Considerando que os idosos formam o grupo com maior risco de desenvolver câncer, essa população precisa ter informações confiáveis e acuradas para prevenir sua ocorrência. Desse modo, torna-se imperativo conhecer como os idosos percebem o câncer. A partir desse conhecimento, é possível prever comportamentos relacionados à saúde, possibilitando a criação de estratégias para educação em saúde e melhoria da prevenção do câncer nesse grupo específico (Braz et al., 2018).

Embora grande parte dos danos relacionados à saúde bucal possa ser prevenida e tratada, a grande maioria das pessoas de idade avançada sequer busca obter o atendimento odontológico (Moreira; De Moraes, 2017). Quando a neoplasia da cavidade bucal é detectada em sua fase inicial, o índice de sobrevida aumenta de forma considerável. O diagnóstico na fase inicial da doença, combinado com um tratamento adequado, parece ser o método mais eficaz para o controle do câncer bucal (Kignel, 2013).

Estudos mais aprofundados das condições de saúde bucal dos idosos, analisando a autopercepção dos indivíduos participantes, o conhecimento e crenças sobre o câncer bucal, necessita ser estimulado. Essa prática deveria ser também estendida para todo atendimento geriátrico, reforçando uma abordagem mais subjetiva, valorizando a assistência odontológica com práticas dialógicas e reconhecendo a realidade vivida em cada município (Moreira; De Moraes, 2017).

Considerando a importância de conhecermos nossa população idosa para que seja possível promover ações de saúde voltadas para suas necessidades, este estudo visou avaliar a condição de saúde bucal dos idosos quanto à presença de lesões potencialmente malignas bem como, verificar o conhecimento e noções acerca destas lesões e avaliar a qualidade de vida dos indivíduos estudados.

\section{Metodologia}

Trata-se de um estudo do tipo observacional transversal no qual indivíduos frequentadores do Parque Municipal do Idoso de Manaus- AM foram avaliados clinicamente quanto a presença de lesões potencialmente malignas na cavidade bucal, grau de conhecimento sobre lesões potencialmente malignas e avaliação da qualidade de vida. A amostra foi calculada com base na prevalência estimada de $18 \%$ de lesões potencialmente malignas em idosos, considerando estudos que determinaram estas prevalências sobre as lesões de interesse para a pesquisa (Bomfim et al., 2008; Martins et al., 2008; Miranda et al., 2011). A pesquisa apresentou população alvo de 2.000 idosos frequentadores do Parque Municipal do Idoso na cidade de Manaus AM, utilizando no seu cálculo erro amostral de 5,0\% e confiança de 95\%, totalizando amostra mínima de 205 idosos, cujo cálculo da amostra foi realizado conforme equação a seguir (Vieira, 2004).

$$
\frac{\mathrm{n}=\quad \mathrm{N} \cdot \mathrm{Z}^{2} \cdot \mathrm{p}(1-\mathrm{p})}{\mathrm{d}^{2} \cdot(\mathrm{N}-1)+\mathrm{Z}^{2} \cdot \mathrm{p}(1-\mathrm{p})}
$$

Onde:

$\mathrm{N}=2000$ (valor da população alvo);

$\mathrm{Z}=1,96$ (valor da distribuição normal para 95\% de confiança);

$\mathrm{p}=0,18$ (valor esperado da prevalência);

$\mathrm{d}=0,05$ (valor do erro amostral). 
A amostra foi composta por idosos frequentadores do Parque Municipal do Idoso situado em Manaus - AM. Os indivíduos foram convidados a participar do estudo, aleatoriamente, através do Termo de Consentimento Livre e Esclarecido TCLE com aprovação do CEP. Em concordância com a Resolução 466/12, do Conselho Nacional de Saúde, principalmente no que concerne à autorização dos participantes, sigilo e confidencialidade, após a assinatura do Termo, os pacientes que obedeceram aos critérios de inclusão e exclusão foram examinados. A pesquisa foi realizada dentro das normas exigidas e aprovada pelo Comitê de Ética em Pesquisa com seres humanos sob o número do protocolo: 20003719.1.0000.5020.

Foram incluídos na pesquisa indivíduos de ambos os sexos, frequentadores regulares de pelo menos uma atividade do Parque do Idoso por semana, com idade igual ou superior a 60 anos, em condições físicas e mentais de responder por sua saúde. Foram excluídos indivíduos incapazes de falar ou se comunicar e idosos com histórico de câncer de boca prévio.

O período de estudo compreendeu de novembro de 2019 a março de 2020. Inicialmente foi aplicado um questionário em forma de entrevista com informações referentes à sua caracterização sociodemográfica, tais como nome, idade, gênero, estado civil, profissão e nível educacional utilizando questões abertas e fechadas (Carvalho et al., 2016; Neto et al., 2017). Após, foi perguntado sobre o grau de satisfação do indivíduo em relação às suas próteses, caracterização da reabilitação protética (tipo de reabilitação removível e o ano de reabilitação), e sobre a qualidade de vida relacionada com a saúde oral utilizando o índice GOHAI (Geriatric Oral Health Assessment Index) (Haikal et al., 2011).

O índice GOHAI é constituído por 12 perguntas, relacionados com a influência dos problemas de saúde oral nas dimensões física, psicossocial, dor ou desconforto. A função física é representada pelo padrão de mastigação, fala e deglutição, a função psicossocial é representada pela preocupação com a saúde bucal, satisfação ou insatisfação com a aparência, autoconsciência sobre a sua saúde bucal e pelo evitar do contato social devido a problemas orais. A dor ou desconforto é representada pelo uso de medicação para aliviar a dor ou desconforto. As opções de resposta variam entre às vezes, sempre e nunca sendo os valores correspondentes a 1, 2 e 3, respectivamente. Para obtenção do índice final, realiza-se a soma simples dos valores, em uma escala de 12 a 36. O índice GOHAI classifica a autopercepção em elevada (34 a 36 pontos), moderada (30 até 33 pontos) e baixa (menos que 30 pontos) (Carvalho et al., 2016).

Após a aplicação do questionário os pacientes foram examinados obedecendo a semiotécnica padrão da Estomatologia. Inicialmente foram examinadas a ATM, percussão do seio frontal e seios maxilares, palpação das cadeias ganglionares cervico-faciais e através da observação de tecidos moles da cavidade bucal, onde foram examinados sistematicamente a semi-mucosa labial, mucosa labial, mucosa jugal, região retromolar, dorso da língua, laterais da língua, ventre lingual, assoalho bucal, palato duro, palato mole, orofaringe e gengivas (Morger et al., 2010).

Nos casos em que foram diagnosticadas lesões cuja indicação de biópsia é expressa, os mesmos foram encaminhados para tratamento. Os casos em que não houve indicação de biópsia o tratamento terapêutico foi instituído. O exame foi realizado por um único examinador especialista em Estomatologia, previamente calibrado utilizando ilustrações de atlas da literatura odontológica com 10 figuras de lesões comuns na cavidade bucal e cancerizáveis. Os achados clínicos foram analisados por um segundo indivíduo também especialista, para que não ocorresse erros no diagnóstico e houvesse um confronto de opiniões. O exame dos pacientes iniciou apenas quando o índice de concordância Kappa demonstrou score satisfatório.

Para a análise dos dados, foram apresentados gráficos e tabelas, onde se calculou as frequências absolutas simples e relativas para os dados categóricos. Na análise das variáveis quantitativas quando aceita a hipótese de normalidade por meio do teste de Shapiro-Wilk foram calculadas a média e o desvio-padrão, no entanto, quando rejeitada a hipótese de normalidade foram calculadas a mediana e os quartis $(25 \%$ e $75 \%)$. Na comparação das medianas dos scores foi aplicado o teste não paramétrico de Mann-Whitney e na prevalência das lesões potencialmente malignas o Intervalo de Confiança ao nível de 95\%IC95\%. Já na comparação das proporç̃̃es foi aplicado o teste exato de Fisher (Vieira, 2004). Foi utilizado o programa Epi Info Versão 7.2.2.6 para Windows, com nível de significância de 5\%. 


\section{Resultados}

Dos indivíduos pesquisados, 111 idosos aceitaram participar, porém apenas 108 atenderam aos critérios de inclusão do estudo, o qual foi suspenso devido a pandemia do COVID 19. Destes 108 participantes avaliados, $91(84,3 \%)$ pertenciam ao gênero feminino e $17(15,7 \%)$ ao gênero masculino. A média de idade dos idosos foi de 72,8 anos (DP $\pm 7,5)$ calculado pelo teste de Shapiro-Wilk com as idades variando entre 60-90 anos. O teste de Shapiro-Wilk é eficiente para diferentes distribuições e tamanhos de amostras, fornecendo o parâmetro valor de prova (valor-p ou significância), que pode ser interpretado como a medida do grau de concordância entre os dados e a hipótese nula $\left(\mathrm{H}_{0}\right)$, sendo que $\mathrm{H}_{0}$ corresponde à distribuição Normal (Lopes, et al., 2013).

Em relação aos dados sociodemográficos dos participantes, constatou-se que a maioria se autodeclarou parda $(54,6 \%)$. Quanto ao estado civil, observou-se que a maior porcentagem era viúva (34,3\%). Referindo-se a escolaridade, foi identificado que a maioria possuía apenas o ensino fundamental $(40,7 \%)$. Quanto à naturalidade dos entrevistados e o grau de ocupação, grande parte dos idosos eram do estado do Amazonas e uma baixa porcentagem trabalhava, conforme a Tabela 1.

Tabela 1. Distribuição segundo a frequência dos dados sociodemográficos dos pacientes amostrados, Manaus - AM.

\begin{tabular}{|c|c|c|c|}
\hline Variáveis $(\mathrm{n}=108)$ & $f_{i}$ & \multicolumn{2}{|c|}{$\%$} \\
\hline \multicolumn{4}{|l|}{ Cor } \\
\hline Branca & & 32 & 29,6 \\
\hline Parda & & 59 & 54,6 \\
\hline Preta & & 17 & 15,7 \\
\hline \multicolumn{4}{|l|}{ Estado civil } \\
\hline Casado & & 33 & 30,6 \\
\hline Separado ou divorciado & & 11 & 10,2 \\
\hline Solteiro & & 24 & 22,2 \\
\hline União estável & & 3 & 2,8 \\
\hline Viúvo & & 37 & 34,3 \\
\hline \multicolumn{4}{|l|}{ Escolaridade } \\
\hline Nunca estudou & & 1 & 0,9 \\
\hline Fundamental & & 44 & 40,7 \\
\hline Médio & & 39 & 36,1 \\
\hline Superior & & 24 & 22,3 \\
\hline \multicolumn{4}{|l|}{ Naturalidade } \\
\hline Amazonas & & 81 & 75,0 \\
\hline Outro Estado & & 27 & 25,0 \\
\hline Trabalha & & 4 & 3,7 \\
\hline
\end{tabular}

$f_{i}=$ frequência absoluta simples.Fonte: Autores (2021).

Foram analisados os resultados quanto ao conhecimento das lesões potencialmente malignas entre os idosos, a presença destas na cavidade bucal e questionamento quanto à influência do álcool e tabaco no desenvolvimento do câncer de boca, esquematizados na Tabela 2. 
Tabela 2. Distribuição segundo a frequência do conhecimento a respeito das lesões potencialmente malignas por meio dos pacientes amostrados, Manaus - AM.

\begin{tabular}{lll}
\hline \hline Variáveis ( $\mathrm{n}=108)$ & $\mathrm{f}_{\mathrm{i}}$ & $\%$ \\
\hline Você sabe o que é câncer de boca & 4 & 3,7 \\
Já ouviu ou leu sobre lesões potencialmente malignas & 42 & 38,9 \\
Apresentou ou apresenta alguma lesão potencialmente maligna & & \\
Sim & 1 & 0,9 \\
Não & 92 & 85,2 \\
$\quad$ Não sabe dizer & 15 & 13,9 \\
Acredita que o uso de álcool e tabaco influenciam no surgimento do câncer de \\
boca & 96 & 88,9 \\
\hline \hline
\end{tabular}

$f_{i}=$ frequência absoluta simples. Fonte: Autores (2021).

Realizaram-se questionamentos sobre a prevenção das lesões potencialmente malignas, diagnóstico precoce, papel do cirurgião dentista ao explicar o que são lesões potencialmente malignas, conhecimento e realização do autoexame bucal na Tabela 3, logo abaixo.

Tabela 3. Distribuição segundo a frequência dos dados sobre a prevenção das lesões potencialmente malignas por parte dos pacientes amostrados, Manaus - AM.

\begin{tabular}{lcc}
\hline \hline Variáveis $(\mathrm{n}=108)$ & $\mathrm{f}_{\mathrm{i}}$ & $\%$ \\
\hline $\begin{array}{l}\text { Concorda que o diagnóstico precoce previne o câncer de boca } \\
\text { O cirurgião dentista alguma vez já explicou o que são lesões potencialmente }\end{array}$ & 103 & 95,4 \\
malignas & 12 & 11,1 \\
Já ouviu falar sobre o autoexame bucal & 47 & 43,5 \\
Sabe como fazer o autoexame bucal & 19 & 17,6 \\
Realiza ou realizou alguma vez o autoexame bucal & 18 & 16,7 \\
\hline \hline
\end{tabular}

$f_{i}=$ frequência absoluta simples. Fonte: Autores (2021).

Quanto à comparação das medianas do escore do índice GOHAI (índice de determinação da saúde oral geriátrica) em relação às lesões potencialmente malignas presentes nos idosos examinados, observou-se que não houve diferença estatisticamente significante entre o índice de GOHAI em pacientes com ou sem lesões potencialmente malignas $(\mathrm{p}=0,739)$ pelo teste não paramétrico de Mann-Whitney. Este teste é utilizado para testar hipóteses envolvendo amostras de dois grupos independentes, como no caso onde se quer comparar dois tratamentos aplicados a dois grupos de pacientes diferentes. O teste, se rejeitar a sua hipótese nula, indica alta possibilidade que exista uma diferença entre as medianas das duas populações de onde as duas amostras foram extraídas (Souza; Castro, 2012).

Dados sobre a distribuição, segundo a prevalência das lesões potencialmente malignas detectadas, como a queilite actínica e a leucoplasia, e das demais lesões identificadas, estão descritos na Tabela 4. 
Tabela 4. Distribuição, na cavidade bucal, das principais lesões identificadas, Manaus - AM.

\begin{tabular}{|c|c|c|}
\hline Variáveis $(\mathrm{n}=108)$ & $f_{i}$ & $\%$ \\
\hline \multicolumn{3}{|l|}{ Lábio } \\
\hline Pigmentação melânica & 51 & 47,2 \\
\hline Grânulos de Fordyce & 36 & 33,3 \\
\hline Queilite Actínica & 1 & 0,9 \\
\hline Outros & 5 & 4,6 \\
\hline \multicolumn{3}{|l|}{ Mucosa labial } \\
\hline Pigmentação melânica & 43 & 39,8 \\
\hline Grânulo de Fordyce & 35 & 32,4 \\
\hline Varicosidades & 13 & 12,0 \\
\hline Outros & 2 & 1,8 \\
\hline \multicolumn{3}{|l|}{ Mucosa jugal } \\
\hline Varicosidades & 46 & 42,6 \\
\hline Linha alba & 32 & 29,6 \\
\hline Grânulo de Fordyce & 23 & 21,3 \\
\hline Leucoedema & 14 & 13,0 \\
\hline Pigmentação melânica & 12 & 11,1 \\
\hline Mancha por amálgama & 2 & 1,8 \\
\hline Outros & 5 & 4,6 \\
\hline \multicolumn{3}{|l|}{ Língua } \\
\hline Saburra & 65 & 60,2 \\
\hline Fissuras & 45 & 41,7 \\
\hline Varicosidades & 37 & 34,3 \\
\hline Nódulos & 2 & 1,8 \\
\hline Outros & 3 & 2,8 \\
\hline \multicolumn{3}{|l|}{ Assoalho bucal } \\
\hline Varicosidades & 75 & 69,4 \\
\hline Outros & 3 & 2,8 \\
\hline \multicolumn{3}{|l|}{ Palato duro } \\
\hline Estomatite protética & 9 & 8,4 \\
\hline Eritema & 7 & 6,5 \\
\hline Pigmentação melânica & 7 & 6,5 \\
\hline Exostose & 4 & 3,7 \\
\hline Petéquias & 3 & 2,8 \\
\hline Hiperemia & 2 & 1,8 \\
\hline Outros & 4 & 3,7 \\
\hline \multicolumn{3}{|l|}{ Palato mole } \\
\hline Estomatite protética & 4 & 3,7 \\
\hline Pigmentação melânica & 2 & 1,8 \\
\hline Outros & 5 & 4,6 \\
\hline
\end{tabular}




\section{Orofaringe}

Hiperemia

Leucoplasia

1

1

Gengiva

Pigmentação melânica

4

Hiperplasia fibrosa inflamatória

Edema

Outros
0,9

0,9

$2 \quad 1,8$

21,8

$3 \quad 2,8$

$\mathrm{f}_{\mathrm{i}}=$ frequência absoluta simples.Fonte: Autores (2021).

Na comparação da presença de lesões potencialmente malignas nos participantes relacionados ao conhecimento sobre lesões potencialmente malignas, verificou-se o domínio e compreensão ao responderem se sabem o que é câncer de boca, se já tinham ouvido ou lido sobre lesões potencialmente malignas, se acreditavam que o uso de álcool e tabaco influencia no surgimento do câncer de boca, detalhados na Tabela 5. Esta comparação foi realizada segundo o teste exato de Fisher, o qual é aplicável a duas pequenas amostras de variáveis com duas categorias (Contador; Senne, 2016).

Tabela 5. Comparação das lesões potencialmente malignas em relação ao conhecimento sobre lesões potencialmente malignas por parte dos pacientes amostrados, Manaus - AM.

Lesões potencialmente malignas

\begin{tabular}{|c|c|c|c|c|c|c|}
\hline \multirow[t]{2}{*}{ Variáveis } & \multicolumn{2}{|c|}{$\begin{array}{l}\operatorname{Sim} \\
(n=2)\end{array}$} & \multicolumn{2}{|c|}{$\begin{array}{l}\text { Não } \\
(n=106)\end{array}$} & \multirow[b]{2}{*}{ Total } & \multirow[b]{2}{*}{$\mathrm{p}^{*}$} \\
\hline & $f_{i}$ & $\%$ & $f_{i}$ & $\%$ & & \\
\hline Sabe o que é câncer de boca & - & - & 49 & 46,2 & 49 & 0,499 \\
\hline $\begin{array}{l}\text { Já ouviu ou leu sobre lesões potencialmente } \\
\text { malignas }\end{array}$ & 1 & 50,0 & 41 & 38,7 & 42 & 0,999 \\
\hline $\begin{array}{l}\text { Acredita que o uso de álcool e tabaco } \\
\text { influenciam no surgimento do câncer de } \\
\text { boca }\end{array}$ & 2 & 100,0 & 94 & 88,7 & 96 & 0,999 \\
\hline
\end{tabular}

$\mathrm{f}_{\mathrm{i}}=$ frequência absoluta simples; * Teste exato de Fisher. Não foi constatada diferença estatística ao nível de $5 \%$ de significância. Fonte: Autores (2021).

\section{Discussão}

A população idosa cresce vertiginosamente no país, que adota o conceito da Organização Mundial da Saúde de idoso como o indivíduo de 60 anos de idade ou mais, se ele residir em países em desenvolvimento. Em 1920, a expectativa de vida era de apenas 35,2 anos e os idosos representavam 4,0\% da população total do país. Com esse perfil, o Brasil tinha para cada 100 crianças (0 a 14 anos), aproximadamente 11 idosos (Miranda et al., 2016). Devido à mudança demográfica do Brasil, a população de idosos tornou-se o novo grupo de risco das neoplasias bucais. De tal modo, a saúde bucal na população idosa deve ser uma prioridade na política de saúde pública (Peralta et al., 2017).

No presente estudo, observou-se disparidade quanto aos gêneros dos participantes. Desses, o gênero feminino predominou com 84,3\% dos entrevistados quando comparado ao masculino, 15,7\%. Pode se dizer que estes dados estão interligados à epidemiologia da doença, comprovadas em outro estudo, na qual há predileção pelo gênero masculino, em uma proporção de 2:1 com o gênero feminino (Furtado et al., 2019). Este fato evidencia que mulheres, quando comparada aos 
homens, procuram mais os serviços de saúde, pois, fazem visitas mais regularmente ao dentista, bem como exames preventivos (Moreira; De Moraes, 2017).

Com relação à escolaridade, apenas $0,9 \%$ nunca estudaram, indicando-se maior porcentagem no grupo que respondeu ter até o ensino fundamental (40,7\%). A baixa escolaridade da população brasileira de 60 anos ou mais é exibido pelos indicadores sociais do Instituto Brasileiro de Geografia e Estatística - IBGE, que revela uma média de 4,2 anos de estudo, com $28,1 \%$ da população possuindo menos de um ano de estudo e apenas 7,2\% possuindo um diploma de graduação (IBGE, 2013). Dessa forma, podemos correlacionar a baixa escolaridade com a possível ausência de acesso às orientações e conhecimentos essenciais à manutenção e cuidados da saúde bucal destes indivíduos.

A informação em saúde tem por objetivo o desenvolvimento de capacidades individuais e coletivas que levam a melhorias na qualidade de vida da população (Oliveira et al., 2020). Dessa forma, ao responderem perguntas voltadas para o conhecimento dos idosos quanto ao câncer de boca e lesões potencialmente malignas, ocorreu outra disparidade. Apenas 3,7\% declararam saber o que é o câncer de boca, apesar de que 38,9\% afirmaram ter ouvido ou lido sobre lesões potencialmente malignas. De fato, em um estudo analisou-se que o baixo conhecimento pode ser influenciado pelos níveis de conhecimentos prévios, pela competência e motivação das pessoas que tiveram acesso a informações relacionadas à saúde (Martins et al., 2020). A falta de informação da população geral e dos profissionais de saúde sobre a prevenção dessa neoplasia, principalmente em indivíduos expostos a fatores de risco, atrasa o diagnóstico dessa neoplasia (Torres; Sbegue; Costa, 2016).

$\mathrm{O}$ uso de tabaco e o consumo de álcool são fatores etiológicos bem estabelecidos para o desenvolvimento das desordens orais potencialmente malignas, mas também há boas evidências de que o risco de progressão está relacionado à prática ou à prática continuada desses hábitos (Speight; Khurram; Kujan, 2018). Dessa forma, quando analisados na pesquisa estes fatores, observou-se que $88,9 \%$ dos entrevistados concordaram que há uma relação sinérgica no uso de álcool e tabaco. Isto também é reconhecido em outro estudo, ao qual relata que dentre os idosos entrevistados os hábitos tabagistas e etilistas foram ausentes na maioria dos idosos (Martins et al., 2020).

As práticas de prevenção e promoção de saúde, que busquem o diagnóstico precoce de lesões suspeitas, possibilitam maiores chances de cura e de aumento da sobrevida dos pacientes, especialmente entre os idosos (Torres; Sbegue; Costa, 2016). Portanto, quanto ao autoexame ser utilizado como uma ferramenta de prevenção, $43,5 \%$ descreveu ter conhecimento. Em contrapartida, uma menor prevalência $(16,7 \%)$ disse ter feito ou fazer o autoexame. Todavia, em uma pesquisa este dado foi arbitrário, pois apenas 31,40\% dos pesquisados relataram ter conhecimento sobre o autoexame, enquanto 68,59\% negaram saber (Moreira; De Moraes, 2017). Já em outro estudo, a maioria das pessoas relataram nunca ter ouvido falar sobre câncer de boca, mas mostraram bastante interesse no assunto e participaram ativamente da prática do autoexame na frente do espelho (Silva et al., 2018).

A maioria dos idosos entrevistados $(95,4 \%)$ declarou que o diagnóstico precoce coopera na prevenção do câncer de boca, sendo relevante dizer que há estudos confirmando que popularizar o conhecimento relativo ao autoexame é incentivar a prevenção do câncer de boca. (Silva et al., 2018; Torres; Sbegue; Costa, 2016; Moreira; De Moraes, 2017). Portanto, a vigilância em saúde e o acompanhamento dos casos suspeitos torna-se uma medida fundamental para o diagnóstico precoce e o pronto tratamento (Casotti et al., 2016). Ações preventivas, diagnóstico precoce e correto tratamento para o câncer de boca são medidas que devem ser tomadas para melhorar os indicadores desse grande problema de saúde pública (Freire et al., 2017).

Por outro lado, apenas $11,1 \%$ dos participantes relataram terem recebido explicações e orientações sobre lesões potencialmente malignas em visita ao cirurgião dentista. Dessa forma, há evidências científicas que reconhecem a importância desse profissional no que se refere à prevenção do câncer bucal, devendo combater este problema na atenção primária, visando o diagnóstico e ao tratamento em estágios iniciais da doença (De Freitas et al., 2020; Peralta et al., 2017; Furtado et al., 2019). De acordo com determinadas pesquisas, a grande maioria dos dentistas não está preparada para realizar a biópsia como uma 
atividade de rotina. Considerando que a maior parte das biópsias dos tecidos orais é realizada em ambulatório, a baixa complexidade tecnológica para realizar o procedimento e sua eficácia para o diagnóstico precoce do câncer de boca, quando diagnosticadas lesões suspeitas, o padrão-ouro para diagnóstico de cânceres orais e lesões potencialmente malignas deve ser o exame histopatológico de espécimes de biópsia cirúrgica. Assim, é possível constatar que a distribuição das doenças orais utilizando biópsias permite uma maior precisão nos dados sobre a saúde bucal de pacientes idosos, especialmente quando se considera lesões malignas e pré-malignas (Noro et al., 2017; Moreira; De Moraes, 2017; Cavalcante et al., 2016).

Foram realizados exames extra e intra-oral em cada participante. Dentre as lesões potencialmente malignas, foram identificados apenas dois casos enquadrados neste grupo de lesões: a queilite actínica e leucoplasia $(1,8 \%)$. É relevante mencionar que em uma determinada pesquisa, os dados coletados foram semelhantes, pois constatou-se que a maioria dos idosos analisados não apresentaram alterações na mucosa (Martins et al., 2020). Já em outra pesquisa, o estudo mostrou uma alta prevalência de câncer oral (10,4\%), sendo ainda maior que a de lesões potencialmente malignas $(7,4 \%)$ (Cavalcante et al., 2016). Dessa forma, pode-se interpretar que estes resultados podem variar de acordo com o local, estilo de vida, hábitos e rotina do idoso. Neste caso, como a pesquisa foi realizada em um Parque do Idoso, os idosos frequentadores recebem orientações para o tratamento da sua saúde bucal a partir de campanhas, projetos e de pesquisas como esta. Referindo-se às outras lesões detectadas e que se definem dentro do padrão da normalidade, as mais significativas e que se destacam como normais na terceira idade foram: varicosidades e saburra lingual.

Existem vários instrumentos utilizados para se fazer um levantamento da saúde bucal e qualidade de vida do idoso, a título de exemplo o GOHAI (Geriatric Oral Health Assessment Index - índice geriátrico para avaliação de saúde bucal). Utilizando este índice para avaliar a autopercepção da saúde bucal, realizaram-se duas medianas dos escores dos participantes, uma entre idosos que indicaram lesão cancerizável, outra entre idosos que não apresentaram tais lesões. Dessas, notou-se que a mediana dos que apontaram lesão resultou em 26, e a do grupo que não teve como resultado 28. De uma forma geral, no grupo estudado, a autopercepção de saúde bucal pode ser considerada "ruim", a partir do índice de GOHAI médio encontrado.

Além disso, a autopercepção encontrada na pesquisa não condiz com dados da literatura, na qual os estudos mostram que os idosos atribuem valores positivos à sua saúde oral, mesmo detectando-se situações clínicas patológicas e prejudiciais (Moreira; De Moraes, 2017). É possível também relacionar esta elevada autopercepção com a baixa escolaridade, visto que tanto neste estudo, como em outros é observado que a maioria dos idosos não chegam a concluir o ensino fundamental, médio ou superior (Martins et al., 2020; Neto et al., 2017; Moreira; De Moraes, 2017). Como consequência da baixa escolaridade, os idosos acabam não possuindo entendimento do que seria uma verdadeira qualidade de vida bucal e não saberiam detectar possíveis lesões em sua cavidade oral.

É importante ressaltar a necessidade de estudos que possam analisar uma possível associação entre os dados coletados no GOHAI com a presença ou não das lesões potencialmente malignas nos idosos. Principalmente nos idosos que veem a sua qualidade de vida oral como boa/ótima, mas que ao exame clínico é detectado uma lesão potencialmente maligna. Investigar as diferentes variáveis que podem influenciar a autopercepção bucal geriátrica e a existência destas respectivas lesões também é uma forma de prevenção ao câncer de boca.

\section{Conclusão}

Com base nos resultados da pesquisa, pôde se observar um baixo grau de conhecimento a respeito das lesões potencialmente malignas bucais, não havendo valores estatisticamente expressivos ao correlacionar a qualidade de vida com a presença destas lesões e a qualidade de vida avaliada no grupo pelo GOHAI teve escore baixo. Os idosos acometidos por lesões potencialmente malignas não souberam afirmar o que é câncer de boca. Metade relataram conhecimento destas lesões e houve unanimidade quanto ao uso de álcool e tabaco serem fatores de risco do câncer de boca. Assim, o desenvolvimento de 
maiores ações de promoção e prevenção da saúde bucal geriátrica, vinculada a esclarecimentos quanto às lesões potencialmente malignas e o câncer de boca, são notavelmente imprescindíveis.

O trabalho foi financiado pela Fundação de Amparo à Pesquisa do Estado do Amazonas - FAPEAM, com número do processo: 062.00999/2019 como resultado de uma pesquisa de iniciação científica. Não houve conflito de interesses entre os autores. Este trabalho recebeu menção honrosa no Congresso de Iniciação Científica - CONIC da Universidade Federal do Amazonas - UFAM no ano de 2020.

\section{Agradecimentos}

Agradecemos o apoio da Universidade Federal do Amazonas - UFAM e PROPESP - Pró-Reitoria de Pesquisa e PósGraduação pela viabilização do projeto de iniciação científica, ao Parque Municipal do Idoso em Manaus pelo acolhimento e atenção com a equipe de pesquisa, à Fundação de Amparo à Pesquisa do Estado do Amazonas - FAPEAM pelo fomento e a colaboração de todos os participantes.

\section{Referências}

Bomfim, I. P. R., Soares, D. G., Tavares, G. R., Santos, R. C., Araujo, T. P., \& Padilha, W. W. N. (2008). Prevalência de lesões de mucosa bucal em pacientes portadores de prótese dentária. Pesquisa Brasileira em Odontopediatria e Clínica Integrada, 8 (1), $117-121$.

Braz, I. F. L., Gomes, R. A. D., Azevedo, M. S. D., Alves, F. D. C. M., Seabra, D. S., Lima, F. P., \& Pereira, J. D. S. (2018). Análise da percepção do câncer por idosos. Einstein (São Paulo), 16 (2), 1-7.

Carvalho, C., Manso, A. C., Escoval, A., Salvado, F., \& Nunes, C. (2016). Autopercepção da saúde bucal em idosos de uma população urbana em Lisboa, Portugal. Revista de Saúde Pública, 50, 53.

Casotti, E., Monteiro, A. B. F., Castro Filho, E. L. D., \& Santos, M. P. D. (2016). Organização dos serviços públicos de saúde bucal para diagnóstico precoce de desordens com potencial de malignização do estado do Rio de Janeiro, Brasil. Ciência \& Saúde Coletiva, 21, 1573-1582.

Cavalcante, A. B. P., de Abrantes, J. G. S., de Sousa, J. N. L., \& Barroso, K. M. A. (2016). Estudo de prevalência de lesões orais malignas ou com potencial de malignização, como estratégia de prevenção do câncer oral: uma revisão de literatura. Revista Saúde \& Ciência Online, 5 (2), $111-127$.

Contador, J. L., \& Senne, E. L. F. (2016). Testes não paramétricos para pequenas amostras de variáveis não categorizadas: um estudo. Gestão \& Produção, 23, 588-599.

De Freitas, C. J. R., Fernandes, M. Â. F., Santos, B. R. M., \& de Souza Rosendo, T. M. S. (2020). Abordagem dos cirurgiões-dentistas da Estratégia Saúde da Família sobre o câncer bucal. Revista da Faculdade de Odontologia-UPF, 25 (2), 198-205.

De Souza Torres, S. V., Sbegue, A., \& Costa, S. C. B. (2016). A importância do diagnóstico precoce de câncer bucal em idosos. Rev Soc Bras Clin Med, 14 (1), 57-62.

Freire, J. C. P., Nóbrega, M. T. C., Freire, S. C. P., \& Ribeiro, E. D. (2017). Incidência de neoplasias malignas orais nas regiões brasileiras. Revista Família, Ciclos de Vida e Saúde no Contexto Social, 5, 141-145.

Furtado, L. S. F. A., Sales, J. L. A. M., Fontes, N. M., de Medeiros Batista, M. I. H., Carvalho, A. A. T., \& Paulino, M. R. (2019). Câncer bucal, desordens potencialmente malignas e prevenção: uma revisão integrativa. Revista Família, Ciclos de Vida e Saúde no Contexto Social, 7 (4), $479-490$.

Haikal, D. S. A., Paula, A. M. B. D., Martins, A. M. E. D. B. L., Moreira, A. N., \& Ferreira, E. F. (2011). Autopercepção da saúde bucal e impacto na qualidade de vida do idoso: uma abordagem quanti-qualitativa. Ciência \& Saúde Coletiva, 16, 3317-3329.

Instituto Brasileiro De Geografia E Estatística (IBGE) Projeção do Brasil por sexo e idade: 2000-2060. http://www.ibge.gov.br/home/e statistica/populacao/projecao_da_populacao/2013/

Instituto Brasileiro De Geografia E Estatística (IBGE) Síntese de indicadores sociais: uma análise das condições de vida da população brasileira 2013. https://biblioteca.ibge.gov.br/visualizacao/livros/liv101629.pdf

Instituto Nacional Do Câncer (INCA) Tipos de câncer 2021. https://www.inca.gov.br/tipos-de-cancer/cancer-de-boca

Instituto Nacional Do Câncer (INCA) Estimativas 2020 Neoplasia maligna da cavidade oral (taxas ajustadas). https://www.inca.gov.br/estimativa/taxasajustadas/neoplasia-maligna-da-cavidade-oral

Kignel, S. (2013). Estomatologia: bases do diagnóstico para o clínico geral. Santos.

Lopes, M. D. M., Castelo Branco, V. T. F., \& Soares, J. B. (2013). Utilização dos testes estatísticos de Kolmogorov-Smirnov e Shapiro-Wilk para verificação da normalidade para materiais de pavimentação. Repositório institucional UFC. 
Research, Society and Development, v. 10, n. 9, e51110918299, 2021

(CC BY 4.0) | ISSN 2525-3409 | DOI: http://dx.doi.org/10.33448/rsd-v10i9.18299

Eleutério Barros Lima Martins, A. M., Silva Souza, J. G., Santos-Neto, P. E., Barbosa Eleutério, N., Sant'Ana Haikal, D., Fagundes Silveira. M., Batista Paula, A. M., Sena Guimarães, A. L., Ferreira, R. C., \& Almeida Pordeus, I. (2020). Prevenção do câncer de boca: acesso a informações e comportamento entre idosos de Montes Claros - MG. Revista Unimontes Científica, 14 (1), 141-153.

Martins, R. B., Giovani, É. M., \& Villalba, H. (2008). Lesões cancerizáveis na cavidade bucal. Rev Inst Ciênc Saúde, 26 (4), 467-76.

Miranda, A. M. O., Ferrari, T. M., \& Calandro, T. L. L. (2011). Queilite Actínica: Aspectos clínicos e prevalência encontrados em uma população rural do interior do Brasil. Saúde e Pesquisa, 4 (1), 67-72.

Miranda, G. M. D., Mendes, A. D. C. G., \& Silva, A. L. A. D. (2016). O envelhecimento populacional brasileiro: desafios e con sequências sociais atuais e futuras. Revista brasileira de geriatria e gerontologia, 19, 507-519.

Moreira, M. E. D. C. C., \& de Moraes, M. S. (2017). Autopercepção da saúde bucal e ciência dos fatores de risco para câncer oral em idosos. Arquivos de Ciências da Saúde, 24 (3), 14-18.

Morger, R., Ramseier, C. A., Rees, T. D., Bürgin, W. B., \& Bornstein, M. M. (2010). Oral mucosal findings related to tobacco use and alcohol consumption: a study on Swiss army recruits involving self-reported and clinical data. Oral health \& preventive dentistry, 8 (2), 143-151.

Neto, B. C. B., Santos, J. L. A., Miranda, S. S., \& de Souza Santos, L. P. (2017). Distribuição, características clínicas e epidemiológicas do câncer bucal no estado da Bahia, 2010-2015. Revista Textura, 10 (19), 138-144.

Noro, L. R. A., Landim, J. R., Martins, M. C. D. A., \& Lima, Y. C. P. (2017). O desafio da abordagem do câncer de boca na atenção primária em saúde. Ciência \& Saúde Coletiva, 22 (5), 1579-1587.

De Oliveira, R. F. R., Ferreira, R. C., da Silva Santos, R. M., Marques, A. P. S. F., Rodrigues, C. A. Q., Nascimento, J. E., \& de Barros Lima, A. M. E. (2012). Idosos: uso dos serviços odontológicos, comportamentos relacionados à saúde e condições subjetivas de saúde. Revista Unimontes Científica, 14 (1), $202-218$.

Peralta, M. M., de Lourdes Caldana, M., da Silva Bastos, R., de Magalhães Bastos, J. R., \& Honório, H. M. (2017). Neoplasias bucais em indivíduos idosos no Brasil: Revisão integrativa. Revista Rede de Cuidados em Saúde, 10 (1).

Silva, M. A., Souza, J. R., Oliveira, M. G., \& Rocha, D. A. P. (2018). Câncer de boca: ação educativa centrada na capacitação para o autoexame. Revista Ciência em Extensão, 14 (1), 116-124.

Souza e Castro, P. A. (2012). Tamanho de amostra e poder para três testes não-paramétricos. Repositório digital UFRGS.

Speight, P. M., Khurram, S. A., \& Kujan, O. (2018). Oral potentially malignant disorders: risk of progression to malignancy. Oral surgery, oral medicine, oral pathology and oral radiology, 125 (6), 612-627.

Vieira, S. (2004). Bioestatística: Tópicos Avançados. Elsevier.

Vieira, A. O. A. (2013). Prevalência de lesões cancerizáveis na mucosa bucal de pacientes portadores de alcoolismo crônico. Relatório de pesquisa UFAM. 